\title{
Disastrous performance of NanoCote/Aqua Based antimicrobial paint in a hospital setting
}

\author{
J.J. Ramsden, ${ }^{1, *}$ M. Reid, ${ }^{2}$ V. Whatley ${ }^{2}$ and S.J. Dancer ${ }^{3,4}$ \\ ${ }^{1}$ Clore Laboratory, University of Buckingham, MK18 1EG, UK \\ ${ }^{2}$ The Royal Wolverhampton NHS Trust, New Cross Hospital, Wolverhampton Road, Heath Town, Wolverhampton WV10 0QP, UK \\ ${ }^{3}$ Department of Microbiology, Hairmyres Hospital, Eaglesham Road, East Kilbride, Glasgow G75 8RG, UK \\ ${ }^{4}$ Department of Applied Sciences, Edinburgh Napier University, Edinburgh EH11 4BN, UK
}

\begin{abstract}
Attempts are being made to incorporate copper into clear varnish in order to coat environmental surfaces in hospitals with the long-term goal of reducing healthcare-acquired infections (HAI). The performance of a nanocopper-containing polyacrylic emulsion, NanoCote HD-WR, on bedrails, footboards, control panels, tables and lockers was evaluated. The paint was applied in four coats using conventional spraying technology according to the manufacturer's instructions to give a total thickness of c. $30 \mu \mathrm{m}$. After overnight curing the "clear" coat appeared rough, with uneven mottling, and remained clearly visible on all surfaces to which it had been applied. The coating demonstrated an unpleasant rubbery feel, engendering complaints from patients. Even the merest contact with water, such as light touching with a damp cloth, caused immediate blanching of the coating and the surface became extremely slippery. Contact with a lightly soiled cloth caused irreversible discoloration of the coating. The physical properties of this coating render it unacceptable for application in hospitals.
\end{abstract}

Keywords: adhesion, cohesion, nanocopper, water resistance

\section{INTRODUCTION}

There is considerable interest in the potential of antimicrobial surfaces in hospitals to reduce hospitalacquired infections (HAI). Two principal questions need to be answered: (1) are inanimate surfaces important reservoirs for microbes? and (2) can antimicrobial surfaces reduce the environmental microbial burden? The answer to (1) is far from clear, with both "yes" [1] and "no" [2] being promulgated. Nevertheless, it has at least been conceded that microbial contamination on surfaces can be, and probably is, transferred to patients via human hands [3] (while noting that well-designed studies demonstrating that point, or its converse, are so far lacking). If so, proper hand hygiene might be the answer, although Dancer has pointed out that it is, practically, easier to improve cleaning than hand hygiene [4], since the latter, more profoundly than the former, involves behavioural change rather than merely setting up a "standard operating procedure" (SOP) or similar. The answer to (2) is more straightforward: the burden can be reduced and quite a few technologies are now available for doing so [5], which might help to overcome some of the difficulties associated with conventional disinfection [6].

Recently, considerable interest has been shown in copper as an antimicrobial material. Appreciation of its

\footnotetext{
* E-mail: jeremy.ramsden@buckingham.ac.uk
}

efficacy probably goes back for several millennia, and quite a few studies have succeeded in demonstrating it more or less quantitatively [7-10]. In a frequently-cited paper, Salgado et al. went as far as asserting that copper surfaces reduce the rate of HAI [11]; the critique of this study [12], which effectively negates its conclusions, is unfortunately much less cited.

Even if the link between antimicrobial activity at a surface and reduction of HAI remains to be convincingly demonstrated (cf. [13]), it is certainly plausible enough to warrant further investigation of the antimicrobial effect of different surfaces. Making hospital artefacts from copper may be expensive, aesthetically questionable and impracticable to retrofit. In order to counter those difficulties, there has been some interest in depositing thin films of copper on surfaces made from other materials (e.g. [14]), but this is obviously only possible for objects small enough to fit inside a vacuum chamber. A more useful approach may be to nanify the copper and incorporate it into a paint or varnish that can be readily applied to existing services. The bactericidal activity of copper nanoparticles has been demonstrated [15] (as well as that of copper oxide [16]). Cometa et al. have shown that this activity is retained upon entrapment of the particles in a hydrogel [17], and similarly with conventional polymers [18]. The effective encapsulation of the copper may, furthermore, diminish possible dermatological problems arising through copper hypersensitivity $[19,20]$. 
In recent years considerable progress has been made towards elucidating the bactericidal mechanism of copper [21-27]. There is no need to discuss it here, ${ }^{1}$ other than to remark that the release of copper ions from the surface is required, hence the encapsulation matrix should be chosen so as to allow their (slow) release.

In this work, copper nanoparticles were incorporated into a polyacrylic varnish, which was then sprayed onto "high-touch" surfaces in a hospital ward with the ultimate aim of evaluating its suitability as an antimicrobial coating.

\section{EXPERIMENTAL}

\subsection{Materials}

The antimicrobial paint was NanoCote HD-WR from Global NanoSciences LLC (Sheridan, Wyoming). It is a water-based polyacrylic emulsion (cf. [29]), containing a small amount of polyurethane. The base material is Aqua D from Aqua Based Technologies (Northvale, New Jersey), to which $0.025 \% \mathrm{w} / \mathrm{w} 5 \mathrm{~nm}$-diameter copper particles is added. ${ }^{2}$

The manufacturers of the paint provided a result of antimicrobial efficacy against methicillin-resistant Staphylococcus aureus (MRSA) obtained from a test carried out according to JI S Z 2801 (equivalent to ISO 22196), except that the coating was allowed to act for only 2 hours before determining the remaining number of viable colonies. The resulting $R$-value was 2.89 (i.e., $99.87 \%$ kill). Our own attempts to replicate this result, with a light initial loading of c. $10 \mathrm{CFU} / \mathrm{cm}^{2}$ (S. aureus), yielded $R=0.83$ (85\% kill) after $2 \mathrm{~h}$ and $R=0.98(90 \%$ kill) after $4 \mathrm{~h}$. In a test conducted for the manufacturers according to the AATCC 147 method, no growth was observed upon contact between the treated (textile) sample and the test organism; no zones of inhibition were present. The samples did not show antibacterial activity against Klebsiella pneumoniae.

Four standard hospital beds in one four-bedded bay on a general medical ward were chosen for the study. The target surfaces were the side rails, the footboard and the control panel for each bed, along with four overbed tables and four bedside lockers. For laboratory testing, new white vitreous ceramic tiles from Vitra Karo San (Istanbul, Turkey) were also used as substrates.

\subsection{Methods}

The selected bay had been deep-cleaned according to the local NHS hospital trust's established protocol, namely general cleaning with Hospec "general surface cleaner" (based on alcohol ethoxylate) followed by steam cleaning and exposure to hydrogen peroxide vapour (HPV). Immediately prior to spraying, all surfaces to be sprayed were wiped with a naphtha-based panel wipe (UN 1268). The temperature in the room was $26 \pm 1{ }^{\circ} \mathrm{C}$ and the relative humidity $50 \pm 5 \%$. The room was ventilated by an open window.

The paint was well mixed and passed through a $250 \mu \mathrm{m}$ paper filter before introducing it into the spray equipment. Two spraying methods were used, giving similar results: high-volume, low pressure (HV-LP) and an ordinary $60 \mathrm{psi}$ compressor, both with a high-definition (HD) spray head.

Spraying was carried out by an experienced sprayer. Four thin coats were applied, with about 15 minutes between each one (after which the sprayer judged the coating to be sufficiently dry), aiming for a final thickness of $30 \mu \mathrm{m}$.

\section{RESULTS}

\subsection{Appearance shortly after spraying}

The paint did not spread to form a uniform surface (Fig. 1). In some cases one had the impression that it was simply not wetting the substrate well (Fig. 2). The paint was prone to run on the bedside rails (Fig. 3). This could probably have been avoided by trial and error to slightly reduce the loading on the curved surfaces. The coating on the ceramic tiles was also quite rough (Fig. 4), which is disadvantageous for hospital use - the declivities will likely provide an easier environment for biofilm to build up [34], and it is difficult to reliably assess bacterial colonization since only the uppermost surfaces will tend to be sampled (e.g., if dipslides are used).

\subsection{Appearance and feel after curing}

The appearance did not change upon curing (Fig. 5). Patches could, however, be found that were relatively uniform and smooth (Fig. 6). According to the manufacturer, the coating requires a curing time of at least 12 hours. $24 \mathrm{~h}$

\footnotetext{
1 See the excellent paper by Hans et al. [28]. The bactericidal mechanism of nanocopper seems to be somewhat different from that of massive copper.

2 Note that at this level of addition, the nanocopper is below regulatory thresholds. Eukaryotic genotoxicity has been demonstrated with copper oxide and copper nanoparticles [30-32], and Fu et al. suggest that the generation of reactive oxygen species by many different kinds of nanoparticles constitutes a general mechanism of nanotoxicity (including bacterial genotoxicity) [33]. However, all these works are concerned with the consequences of the nanoparticles themselves penetrating into the cells. In the present case, the nanoparticles are encapsulated within the acrylic polymer and the antimicrobial action is presumed to result from the release of copper ions; genotoxicity and mutagenicity have not been found to be the primary antimicrobial mechanism of copper ions $[9,26]$.
} 


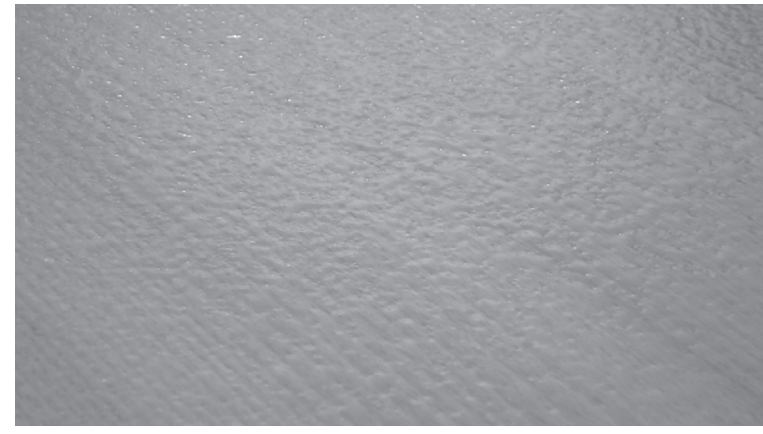

Figure 1. A laminated wood bedside table coated with NanoCote HD-WR (before curing).

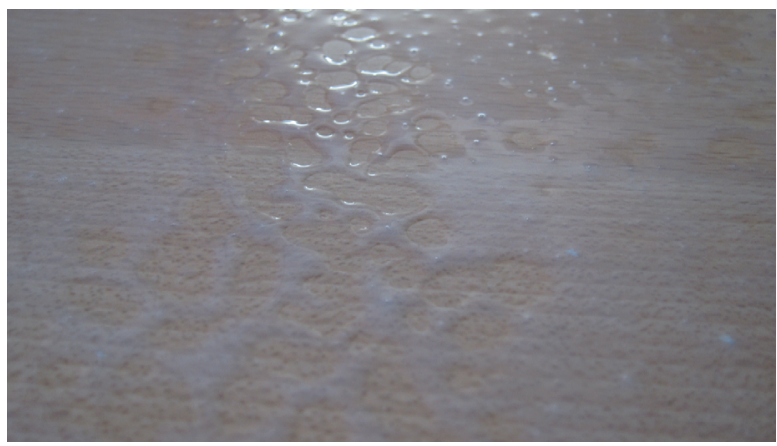

Figure 2. A laminated wood bedside table coated with NanoCote HD-WR (before curing). Close-up view showing a particularly poorly wetted area.

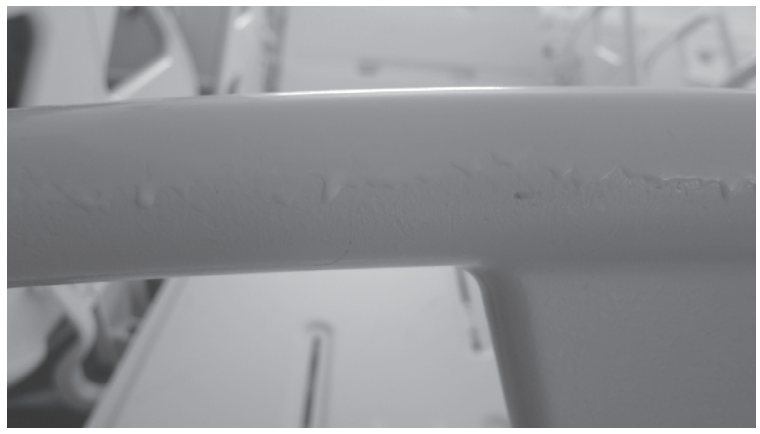

Figure 3. A bedside rail coated with NanoCote HD-WR (before curing).

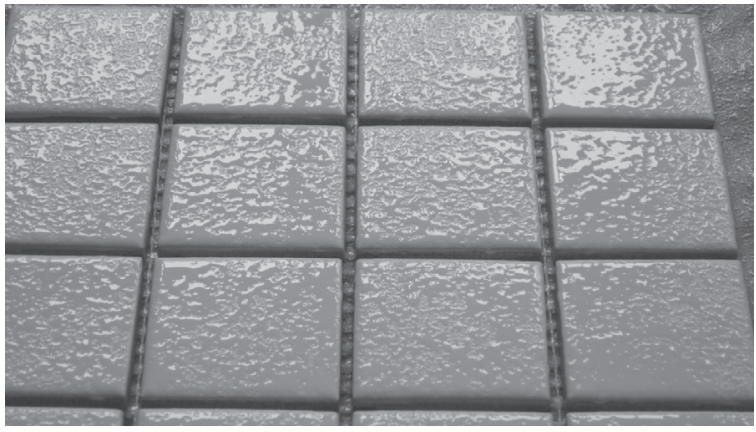

Figure 4. Vitreous ceramic tiles coated with NanoCote HD-WR (before curing). after coating it had a distinctly tacky feel, which was undiminished even 3 weeks after coating. Blowing hot $\left(>200{ }^{\circ} \mathrm{C}\right.$ ) air over the coating removed the tacky feeling, but a few minutes after the hot air current ceased the tackiness returned.

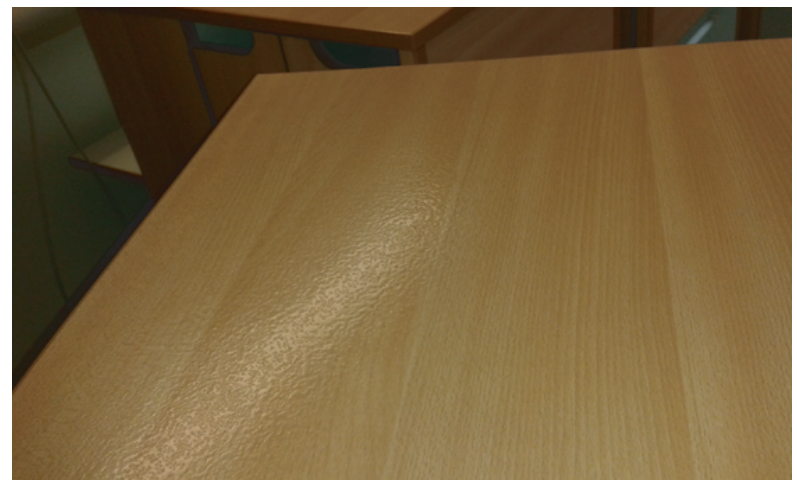

Figure 5. A laminated wood bedside table coated with NanoCote HD-WR (after curing).

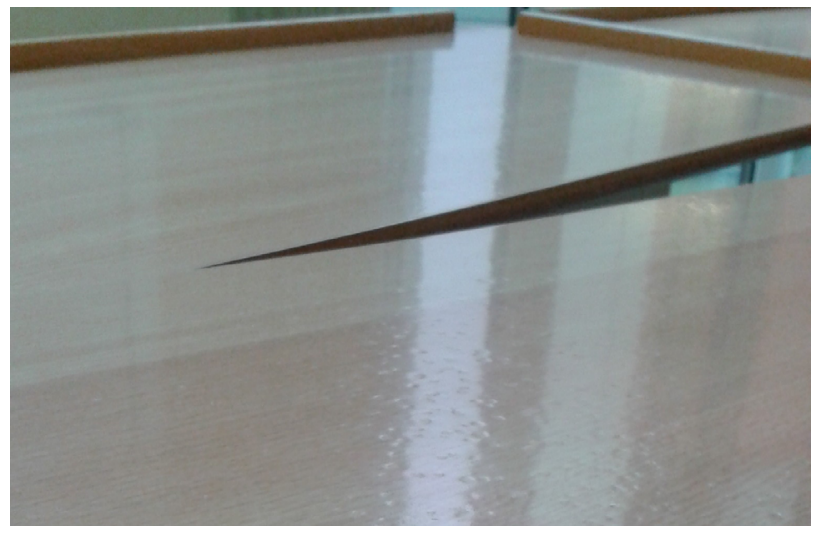

Figure 6. Laminated wood bedside tables coated with NanoCote HD-WR (after curing), showing a somewhat smoother final finish, but cratering is still obviously present.

\subsection{Blanching upon wetting}

Spilling water on the coated and cured surface, or briefly touching it with a moist cloth, resulted in immediate blanching (Fig. 7). As the water slowly evaporated, the original appearance was restored.

\subsection{Coefficients of friction in the wet and dry states}

Three weeks after coating, coefficients of static friction were measured by placing a $20 \mathrm{~g}$ cylindrical brass weight (diameter $14 \mathrm{~mm}$ ) on the coated tiles and measuring the angle at which the weight started to slip. During the measurements the room temperature was $19.4 \pm 0.5^{\circ} \mathrm{C}$ and the relative humidity $57 \pm 2 \%$. The results are given in Table 1 . The same tiles were then moistened by gently wiping three times with the pressure of $200 \pm 30 \mathrm{~g}$ with a wet cotton rag and the measurements were repeated. 


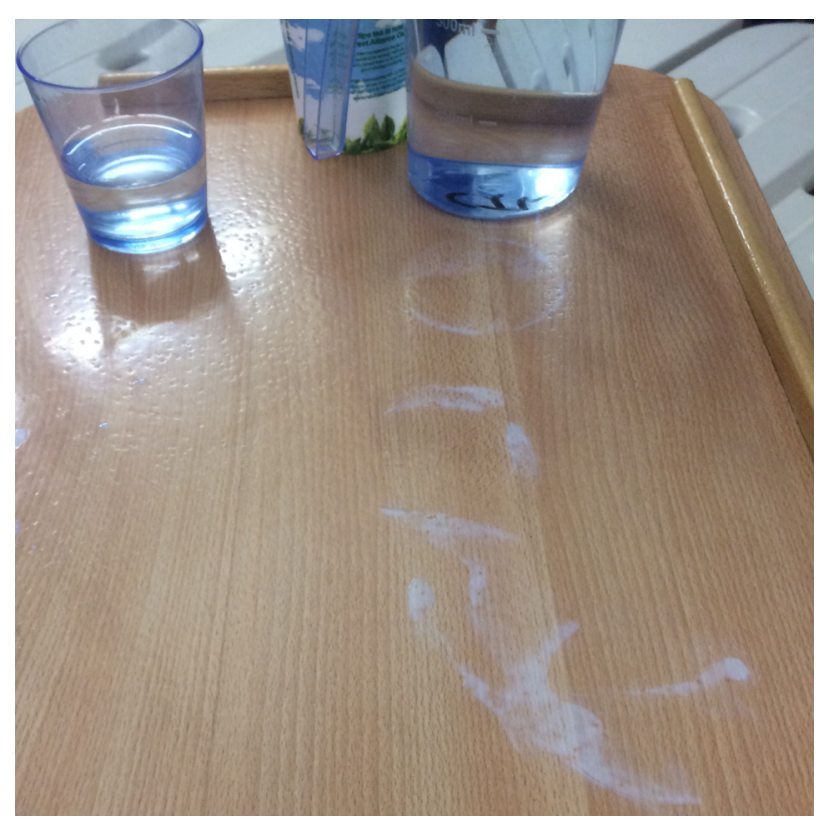

Figure 7. A laminated wood bedside table coated with NanoCote HD-WR after curing, on which some water had made contact with the surface.

Table 1. Coefficients of static friction of a cured NanoCote HD-WR coating on ceramic tiles.

\begin{tabular}{lcc}
\hline & Dry & Wet \\
\hline $\begin{array}{l}\text { Coefficient of } \\
\text { static friction }\end{array}$ & $0.012 \pm 0.001$ & $0.025 \pm 0.004$ \\
\hline
\end{tabular}

\subsection{Durability of the coating}

After curing, the coating seemed to be reasonably adherent, but was nevertheless soft enough to be removed by a fingernail. After wetting it could be scratched off very easily (i.e., loss of adhesion). Indeed, during the threefold wiping (\$3.4) some of the coating flaked off onto the rag (i.e., loss of cohesion). This behaviour did not noticeably change during three weeks after coating. Furthermore, the tackiness of the coating makes it prone to take up dirt from the environment (e.g., if lightly rubbed with a slightly dirty cloth).

\section{DISCUSSION}

The main problems with the coating are the uneven surface, its persistently tacky feel, and the rapid softening upon wetting. As a result, the coating is completely unsuitable for use in the hospital environment, at least for any surface that is touched and, hence, regularly cleaned. It could be used for ceilings but there is little evidence that ceilings are reservoirs for hospital pathogens.

When wetted, the coating immediately feels very slippery (e.g., when a finger lightly touches and is slid horizontally over it). This behaviour is belied by the significant increase in the coefficient of static friction for brass upon wetting.

We cannot exclude the possibility that very careful optimization of coating conditions might result in a better finish of the coating. For example, roughening a laminated wood surface (e.g., by sanding) typically improves wetting and, hence, adhesion of a water-based coating. Preapplication of a customized primer might also help. Nevertheless, given the extremely limited access to ward bays for such interventions, every additional procedure makes the whole less attractive, and any set of procedures taking longer than 24 hours from start to finish is impracticable. Similarly, great sensitivity to the coating conditions increases the risk of failure; there is little doubt that a sprayer could become highly expert in applying this particular coating, but if he or she were unavailable for a particular job, the end result might be substandard. In any case, it is by no means clear that the poor quality of the coating was due to a faulty application technique. The manufacturers initially suggested electrostatic spraying, but presumably they were thinking of uncoated metal substrates. Some attempts were made to apply the coating electrostatically in the laboratory but the coating quality tended to be even worse.

It is an interesting question whether the poor performance of the coating is due to the presence of the copper nanoparticles. Their mass fraction is indeed very low, but they are also very small and, on average, the distance between them is $\sim 2 \mu \mathrm{m}$. The manufacturers are better placed to answer this question empirically by carrying out equivalent procedures on material lacking the nanocopper loading.

It is still a moot point whether nanoparticles embedded in an organic polymer film is an effective way of delivering microbicidal copper. The limited data so far available suggest only modest antimicrobial action. Mathews et al. emphasized the need of the bacteria to be in contact with the copper if killing is to occur [27]. Nevertheless, the polyacrylic material used in NanoCote HD-WR may be sufficiently permeable to allow copper to be present at the surface on which the bacteria will arrive.

\section{CONCLUSIONS}

Clearly this particular paint formulation had problems. Such unexpected behaviour had not been predicted by the manufacturers. Nevertheless, the failure provided some very useful lessons applicable to other coatings of the same generic type, suggesting that any proposed use of antimicrobial films in the form of paint or varnish should be approached cautiously. The following points seem to be particularly pertinent: 
1. Retrofitting demands that the appearance of coated artefacts remains unchanged. It is difficult to make a polymer film thinner than $20-30 \mu \mathrm{m}$, hence even if its optical attributes are "perfect" (i.e., colourless, transparent and smooth) it is likely to be visible, especially on windows, mirrors and display screens.

2. The way a coating feels to the touch is as important an attribute as any other. It seems that tactile properties were not considered by the manufacturers. Hospital patients may be particularly sensitive to the way their bed rails feel when they grab them (since they are the primary interface with the outer world).

3. The cratered appearance is visually unattractive and makes it difficult to clean the surfaces, let alone sample them for microbial contamination.

4. Most varnishes will blanch reversibly when water is placed on them. This particular coating blanched particularly rapidly and underwent a dramatic change in the way it felt to the touch. Again, hospital patients (and their visitors) notice the appearance of their immediate environment; they may critically judge anything that seems to be dirty or defective. There is always a slight risk that a glass of drinking water is upset on a bedside table, and all high-touch surfaces are typically wiped daily with a moist, detergent-loaded cloth. Any coating that changes its attributes when moistened, even if only transiently, is not acceptable in busy clinical settings.

5. Almost inevitably when applying a coating by spraying, droplets diffuse around and land on places other than the primary target. This may cause unexpected malfunctions. For example, some varnish was inadvertently deposited on the piston of the hydraulic bed-raising mechanism, causing it to stick. All non-target surfaces could be meticulously masked, but this would make the whole application operation very cumbersome.

In conclusion, if any film-forming material is being contemplated as an antimicrobial coating, its generic properties (i.e., everything apart from its antimicrobial action, which presumably will have already been thoroughly established in the laboratory) should be carefully tested beforehand on every kind of surface onto which its application is envisaged. Given the typically brief time (one day) available for retrofitting, coatings requiring lengthy curing (longer than overnight) are unsuitable. Our experience suggests that polymer-based film-forming coatings are generally unsuitable for use in the hospital environment. Photocatalytic antimicrobial coatings $[5,35]$ might be more practically useful, because they do not require organic polymers and are much thinner (about $1 \mu \mathrm{m}$ ).

Products are sometimes placed on the market following successful laboratory trials of the main attribute (e.g., antimicrobial efficacy), the manufacturers being reluctant to undertake further trials in a real-world environment because "we know it works". In the study reported here, numerous pitfalls revealed themselves that would not normally emerge in a laboratory study.

\section{ACKNOWLEDGMENTS}

We thank the Royal Wolverhampton NHS Trust staff from the Domestic Services department and the Cardiothoracic ward in which the evaluation of the coating took place for their kind assistance. We also thank Global Nanosciences LLC (Sheridan, Wyoming) for having generously provided the NanoCote HD-WR paint, and for some discussion about the possible reasons for the results obtained; and Andrea Clemente (University of Loughborough) for having undertaken some investigations of the microbiological activity of the NanoCote HD-WR coating in his laboratory.

\section{REFERENCES}

1. Rutala, W.A. and Weber, D.J. The benefits of surface disinfection. Am. J. Infection Control 32 (2004) 226-231.

2. Dettenkofer, M., Wenzler, S., Amthor, S., Antes, G., Motschall, E. and Daschner, F. Does disinfection of environmental surfaces influence nosocomial infection rates? Asystematic review. Am. J. Infection Control 32 (2004) 84-89.

3. Dettenkofer, M. and Spencer, R.C. Importance of environmental the contamination-a critical view. $J$. Hospital Infection 65 Suppl. 2 (2007) 55-57.

4. Dancer, S.J. Importance of the environment in meticillinresistant Staphylococcus aureus acquisition: the case for hospital cleaning. Lancet Infectious Diseases 8 (2008) 101-113.

5. Page, K., Wilson, M. and Parkin, I.P. Antimicrobial surfaces and their potential in reducing the role of the inanimate environment in the incidence of hospital-acquired infections. J. Mater. Chem. 19 (2009) 3819-3831.

6. Dancer, S.J. The role of environmental cleaning in the control of hospital-acquired infection. J. Hosp. Infect. 73 (2009) 378-385.

7. Noyce, J.O., Michels, H. and Keevil, C.W. Potential use of copper surfaces to reduce survival of epidemic meticillinresistant Staphylococcus aureus in the healthcare environment. J. Hospital Infection 63 (2006) 289-297.

8. Casey, A.L., Adams, D., Karpanen, T.J., Lambert, P.A., Cookson, B.D., Nightingale, P., Miruszenko, L., Shillam, R., Christian, P. and Elliott, T.S.J. Role of copper in reducing hospital environment contamination. J. Hospital Infection 74 (2010) 72-77.

9. Grass, G., Rensing., C. and Solioz, M. Metallic copper as an antimicrobial surface. Appl. Environ. Microbiol. 77 (2011) 1541-1546.

10. Wheeldon, L.J., Worthington, T., Lambert, P.A., Hilton, A.C., Lowden, C.J. and Elliott, T.S.J. Antimicrobial efficacy of copper surfaces against spores and vegetative cells of Clostridium difficile: the germination theory. $J$. Antimicrobial Chemotherapy 62 (2008) 522-525. 
11. Salgado, C.D., Sepkowitz, K.A., John, J.F., Cantey, R., Attaway, H.H., Freeman, K.D., Sharpe, P.A., Michels, H.T. and Schmidt, M.G. Copper surfaces reduce the rate of healthcare-acquired infections in the intensive care unit. Infect. Control Hosp. Epidemiol. 34 (2013) 479-486.

12. Harbarth, S.J., Maiwald, M. and Dancer, S. The environment and health-care-acquired infections: why accurate reporting and evaluation of biological plausibility are important. Infect. Control Hosp. Epidemiol. 34 (2013) 996-997; Salgado, C.D., Sepkowitz, K.A., John, J.F., Cantey, R., Attaway, H.H., Freeman, K.D., Sharpe, P.A., Michels, H.T. and Schmidt, M.G. Reply to Harbarth et al. Infect. Control Hosp. Epidemiol. 34 (2013) 997-999.

13. Borkow, G. (ed.). Use of Biocidal Surfaces for Reduction of Healthcare Acquired Infections. Springer (2014).

14. Varghese, S., ElFakhri, S.O., Sheel, D.W., Sheel, P., Bolton, F.J .E. and Foster, H.A. Antimicrobial activity of novel nanostructured $\mathrm{Cu}-\mathrm{SiO}_{2}$ coatings prepared by chemical vapour deposition against hospital-related pathogens. AMB Express 3 (2013) 53.

15. Zhang, Y., Zhu, P., Li, G., Wang, W., Chen, L., Lu, D.D., Sun, R., Zhou, F. and Wong, C. Highly stable and re-dispersible nano $\mathrm{Cu}$ hydrosols with sensitively size-dependent catalytic and antibacterial activities. Nanoscale 7 (2015) 13775-13783.

16. Ren, G., Hu, D., Cheng, E.W.C., Vargas-Reus, M.A., Reip, P. and Allaker, R.P. Characterization of copper oxide nanoparticles for antimicrobial applications. Intl $J$. Antimicrobial Agents 33 (2009) 587-590.

17. Cometa, S., Iatta, R., Ricci, M.A., Ferretti, C. and de Giglio, E. Analytical characterization and antimicrobial properties of novel copper nanoparticle-loaded electro synthesized hydrogel coatings. J. Bioactive Compatible Polymers $\mathbf{2 8}$ (2013) 508-522.

18. Palza, H. Antimicrobial polymers with metal nanoparticles. Intl J. Molec. Sci. 16 (2015) 2099-2116.

19. Hostynek, J.J. and Maibach, H.I. Copper hypersensitivity: dermatologic aspects - an overview. Rev. Environ. Health 18 (2003) 153-183.

20. Fage, S.W., Faurschou, A. and Thyssen, J.P. Copper hypersensitivity. Contact Dermatitis 71 (2014) 191-201.

21. Warnes, S.L., Green, S.M., Michels, H.T. and Keevil, C.W. Biocidal efficacy of copper alloys against pathogenic enterococci involves the degradation of genomic and plasmid DNA. Appl. Environ. Microbiol. 76 (2010) 5390-5401.

22. Warnes, S.L. and Keevil, C.W. Mechanism of copper surface toxicity in vancomycin-resistant enterococci following wet or dry surface contact. Appl. Environ. Microbiol. 77 (2011) 6049-6059.

23. Warnes, S.L., Caves, V. and Keevil, C.W. Mechanism of copper surface toxicity in Escherichia coli O157:H7 and
Salmonella involves immediate membrane depolarisation followed by a slower rate of DNA destruction which differs from that observed for Gram-positive bacteria. Environ. Microbiol. 14(2012) 1730-1743.

24. Quaranta, D., Krans, T., Espírito Santo, C., Elowsky, C.G., Domaille, D.W., Chang, C.J. and Grass, G. Mechanisms of contact-mediated killing of yeast cells on dried metallic copper surfaces. Appl. Environ. Microbiol. 77 (2011) 416- 426.

25. Espírito Santo, C., Lam, E.W., Elowsky, C.G., Quaranta, D., Domaille, D.W., Chang, C.J. and Grass, G. Bacterial killing by dry metallic copper surfaces. Appl. Environ. Microbiol. 77 (2011) 794-802.

26. Espírito Santo, C., Quaranta, D. and Grass, G. Antimicrobial metallic copper surfaces kill Staphylococcus haemolyticus via membrane damage. MicrobiologyOpen 1 (2012) 46-52.

27. Mathews, S., Hans, M., Mücklich, F. and Solioz, M. Contract killing of bacteria on copper is suppressed if bacterial-metal contact is prevented and is induced on iron by copper ions. Appl. Environ. Microbiol. 79 (2013) 2605-2611.

28. Hans, M., Mathews, S., Mücklich, F. and Solioz, M. Physicochemical properties of copper important for its antibacterial activity and development of a unified model. Biointerphases 11 (2016) 018902.

29. Désor, U., Krieger, S., Apitz, G. and Kuropka, R. Waterborne acrylic dispersions for industrial wood coatings. Surf. Coatings Intl 10 (1999 488-496.

30. Alarifi, S., Ali, D., Verma, A., Alakhtani, S. and Ali, B.A.. Cytotoxicity and genotoxicity of copper oxide nanoparticles in human skin keratinocytes. Intl J. Toxicol. 32 (2013) 296-307.

31. Semisch, A., Ohle, J., Witt, B. and Hartwig, A. Cytotoxicity and genotoxicity of nano- and micro-particulate copper oxide: role of solubility and intracellular bioavailability. Particle Fibre Toxicol. 11 (2014) 10

32. Nagaonkar, D., Shende, S. and Rai, M. Biosynthesis of copper nanoparticles and its effect on actively dividing cells of mitosis in Allium cepa. Biotechnol. Progr. 31 (2015) 557-565.

33. Fu, P.P., Xia, Q., Hwang, H.-M., Ray, P.C. and Yu, H. Mechanisms of nanotoxicity: Generation of reactive oxygen species. J. Food Drug Anal. 22 (2014) 64-75.

34. Vickery, K., Deva, A., Jacombs, A., Allan, J., Valente, P. and Gosbell, I.B. Presence of biofilm containing viable multiresistant organisms despite terminal cleaning of clinical surfaces in an intensive-care unit. J. Hospital Infection 80 (2012) 52-55.

35. Ramsden, J.J. Photocatalytic antimicrobial coatings. Nanotechnol. Perceptions 11 (2015) 146-168. 